\title{
Diffuse Neurofibroma of Face: A Rare Case Report
}

\author{
Selva Ganesh S. \\ Assistant Professor, Department of Oral Pathology and Microbiology, Faculty of Dentistry, Meenakshi Academy of \\ Higher Education and Research, Meenakshi Ammal Dental College and Hospital, Chennai, India
}

\begin{abstract}
A case of a 17-year old male who presented with a chief complaint of unesthetic facial appearance due to malformed nose from the time of birth with a history of surgical correction for nasal deformity at the age of 11 years. Microscopy revealed a diagnosis of diffuse neurofibroma with areas showing nerve fibers with wavy nuclei.
\end{abstract}

Keywords: Unaesthetic face, diffuse neurofibroma, case report.

\section{Introduction}

Neurofibromas generally assume three growth patterns: localized, diffuse, or plexiform. Diffuse neurofibroma is not a common variant of neurofibroma, children and young adults being most commonly affected, head and neck being the common sites of involvement. Upon histological examination it appears similar to conventional neurofibromas except for a few unique features. We report a case of a 17 -year old male who presented with a chief complaint of unesthetic facial appearance due to malformed nose from the time of birth this case is reported here because of its unique and rare nature.

Case Report: A 17-year-old boy presented with a chief complaint of unesthetic facial appearance with malformed nose from birth along with swelling of right cheek region. The malformation and swelling was initially small and has attained the current size in a gradual manner. Patient has congenital loss of eye which was treated when the patient was 10 years old. From birth

\section{Corresponding Author:}

\section{Selva Ganesh S.}

Assistant Professor, Department of Oral Pathology and Microbiology, Faculty of Dentistry, Meenakshi Academy of Higher Education and Research, Meenakshi Ammal Dental College and Hospital, Chennai, India e-mail: drselva.oralpathology@madch.edu.in the nose had three nostrils. History of surgical correction for nasal deformity at the age of 11 years but no record of previous surgery was available with patient. Post auricular cartilage was taken for nasal reconstruction.

On clinical examination bifid right nostriland presence of left eye prosthesis was seen. Diffuse swelling of right infraorbital region measuring upto $7 \times 3 \times 5 \mathrm{~cm}$. Swelling was soft in consistency. Scar on right cheek and scar on the midline of nose was noticed (figure 1). The swelling involved only the skin and underlying bone appeared normal upon palpation. No café-au-lait macules, lisch nodules or freckles were seen anywhere in the body.

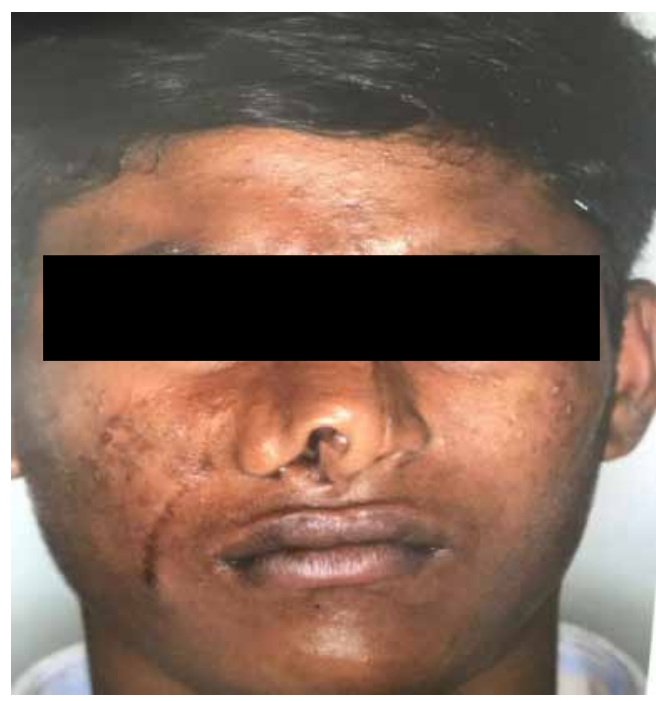

Fig. 1: Diffuse swelling of right infra-orbital region, bifid right nostril and scar on right cheek and scar on the midline of nose. 
No abnormalities were detected in OPG and CT except for nasal polyp. An incisional biopsy was planned and during surgery some cystic fluid or pus discharge from perialar region was observed. Provisional diagnosis of neurofibroma was given by the surgeon. The tissue submitted for biopsy was greyish yellow soft tissue specimen measuring about approximately $(15 \times 32 \times 35)$ $\mathrm{mm} 3$ (figure 2). The tissue was grossed into three bits, each of the cut surface exhibited solid gray white areas intermixed with gelatinous areas (figure 3 ).

The microscopic examination revealed a soft tissue section showing loosely arranged fibrocollagenous tissue with areas showing nerve fibers with wavy nuclei and arranged in fasicles in varying sizes and shapes. These nerve fibers were also seen spread in between the muscle fibers in many areas(figure 4). Less cellular hyalinized regions made up of thin, wispy and loosely arranged cells separated from each other with few microcystic spaces resembling areas known as Antoni $\mathrm{B}$ regions were seen (Figure5). Chronic inflammatory cell infiltrate consisting of lymphocytes, plasma cells and predominantly mast cells were seen with adipocytes and ecstatic vessels. A cystic space lined by epidermis made up of keratinized stratified squamous epithelium in varying thickness with numerous sebaceous glands and other skin adnexal structures like hair follicles and sweat glands were noticed (Figure 6).

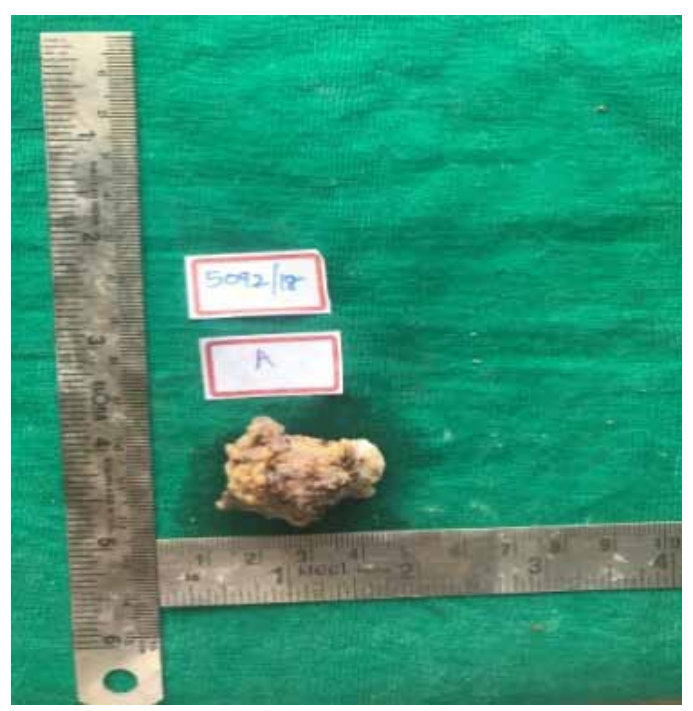

Fig. 2 Greyish yellow soft tissue specimen

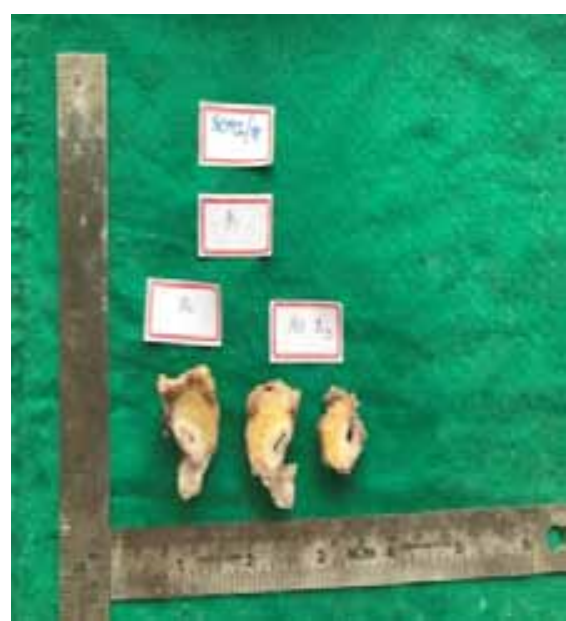

Fig. 3 Cut surfaces exhibiting solid gray white areas intermixed with gelatinous areas

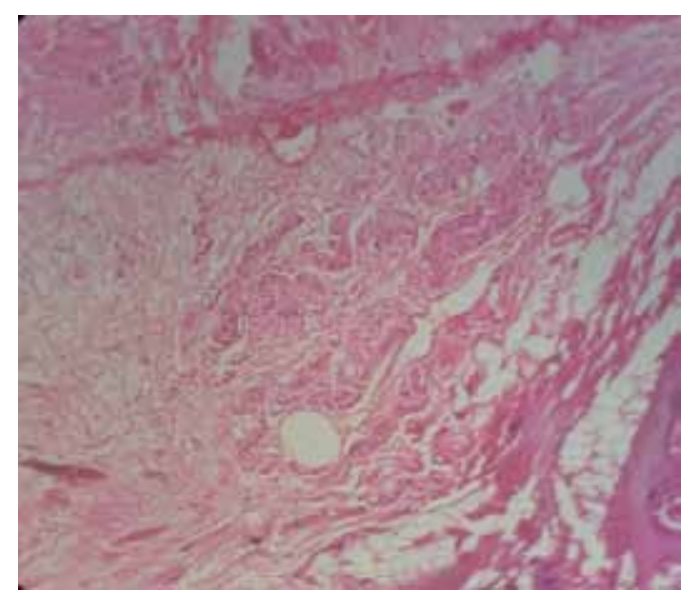

Fig. 4H\&E section under 40x view showing nerve fibers with wavy nuclei and arranged in fasicles in varying sizes and shapes

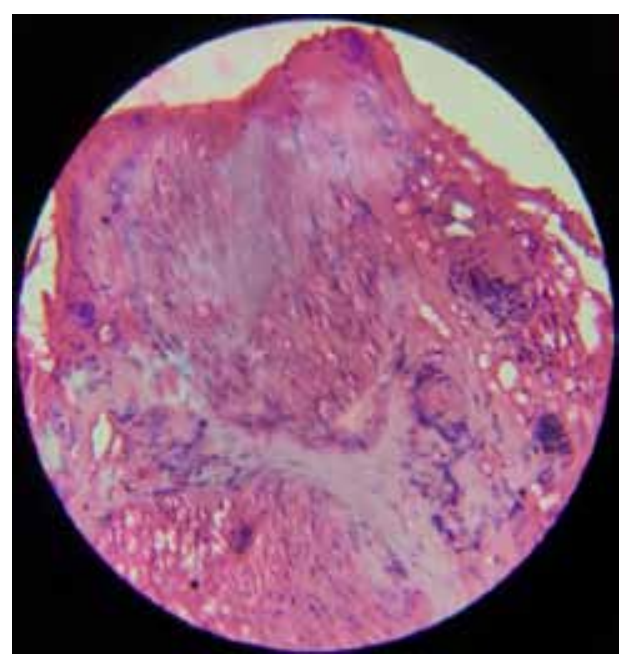

Fig. 5H\&E section under 40x view showing, wispy and loosely arranged cells separated from each other with few microcystic spaces resembling Antoni $B$ regions. 


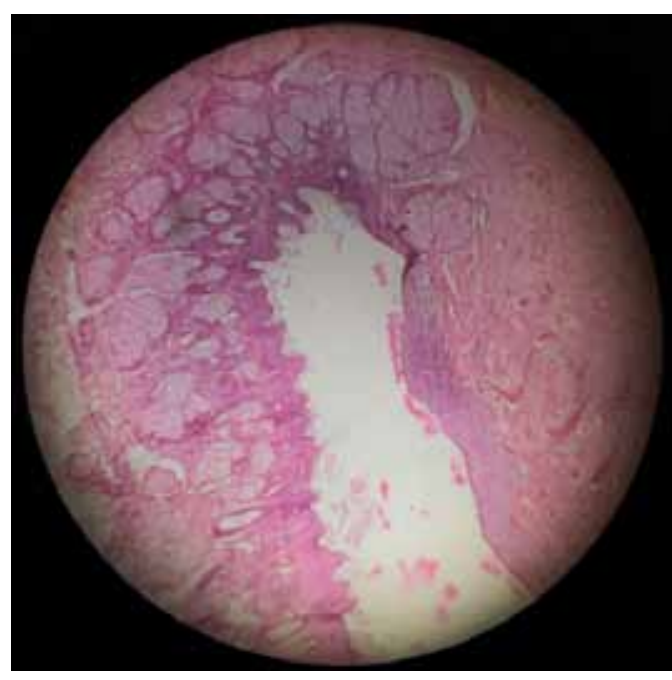

Fig. 6H\&E section under $4 x$ view showing cystic space lined by epidermis made up of keratinized stratified squamous epithelium in varying thickness with numerous sebaceous glands and other skin adnexal structures

\section{Discussion}

A benign peripheral nerve sheath tumor arising from a mixture of Schwann cells and perineural fibroblasts is called as neurofibroma. It can be present either as a solitary lesion or can be associated with neurofibromatosis. According to their growth pattern they can be classified into localized, diffuse, or plexiform ${ }^{[1]}$.

Diffuse neurofibromsa are not common but distinctive type of neurofibroma that is recognized as a separate entity from 1997. It mostly involves the skin and the subcutaneous tissues occurring primarily in $10^{\text {th }}$ and $30^{\text {th }}$ decade of life in either sex ${ }^{[2]}$. They have a slight predilection for trunk, head and neck and the limbs and may become very large as seen in our case. More than $90 \%$ of diffuse neurofibromas are isolated lesions and about $10 \%$ are associated with neurofibromatosis type ${ }^{[3]}$. Diffuse neurofibromas are poorly circumscribed non-encapsulated tumors and typically involve the subcutaneous tissue down to the level of the fascia. As seen in our case the diffuse variant is usually ill-defined and infiltrate the dermis and connective tissue sparing the skin appendages ${ }^{[3,4]}$.

Except for nasal polyp there were no abnormalities seen in our case but generally diffuse neurofibromas erode through the skeletal structures and shows an intracranial extension. These bone deformities can be seen on OPG or computed tomography scans ${ }^{[1,6,8,9,10]}$. The characteristic MR pattern of a diffuse neurofibroma is linear or reticular strands of intermediate signal intensity in the subcutaneous fat, which indicates infiltration of the tumor components of neurofibromatous tissue, interposed collagen, and ectatic vessels ${ }^{[11]}$.

Although benign, these tumors are prone to significant neovascularisation.. Troublesome hemorrhage often occurs during the excision of the neurofibromatous tissue which did not happen in the present case. Hypotensive anesthesia has proven to be effective in decreasing intraoperative bleeding. Because the vessels in tumor or skin lesions are abnormal, incisions must be made on normal skin if possible, which provides better bleeding control ${ }^{[1,6,7]}$.

Histologically, it is characterized by a diffuse replacement of the dermis and subcutis by interlacing bundles of spindle-shaped cells with round or fusiform buckled nuclei and eosinophilic cytoplasm within a loose matrix of fine fibrillary collagen ${ }^{[5]}$, such wavy nuclei of nerve fibers were seen in this case. Though it is infiltrative there is no destruction that will be evident in this histological type instead it envelops the structures like skin adnexal structures, adipose tissue and muscle fibers. Meissner's bodies are characteristic feature of diffuse neurofibroma but rarely they can be absent as reported in this case ${ }^{2}$.

Surgical excision of the tumor is planned only when the functional need is compromised but complete removal is rarely achieved because of the extensive nature and multi centricity of the tumor. Radical surgery is reserved for instances of malignant transformations. However, malignant transformation rarely occurs in the diffuse neurofibroma ${ }^{[1,8,9,12]}$.

\section{Conclusion}

Care must be given during examination and diagnosis of solitary diffuse neurofibroma. Depending on the site of involvement preoperative angiogram is preferred to avoid troublesome hemorrhage during excision. A yearly follow-up of the case is recommended as there is possibility of the potential development of neurofibromatosis and recurrence of the lesion though the malignant transformation is rare.

\section{Ethical Clearance: NA}

Source of Funding: Meenakshi Academy of Higher Education and Research, Chennai, India

Conflict of Interest: NA 


\section{References}

1. Weiss SW. Benign tumors of peripheral nerves. Enzinger and Weiss's soft tissue tumors. 2001:1111-207..

2. Macias VC, Rafael M, Fernandes C, Rosa JC. Diffuse neurofibroma-An uncommon cause of alopecia. Anais brasileiros de dermatologia. 2013 Dec;88(6):166-9..

3. Van Zuuren EJ, Posma AN. Diffuse neurofibroma on the lower back. Journal of the American Academy of Dermatology. 2003 Jun 1;48(6):93840.

4. Khan AK, Deb S, Ray DK, Mandal S, Mukhopadhaya S, Mandal S. Diffuse neurofibroma of scalp. Neurology India. 2002 Oct 1;50(4):516.

5. Chander V, Rao RS, Sekhar G, Raja A, Sridevi M. Recurrent diffuse neurofibroma of nose associated with neurofibromatosis Type 1: a rare case report with review of literature. Indian Journal of Dermatology. 2015 Nov;60(6):573.

6. Chowdary RP, Little BW. Large vascular plexiform neurofibroma of scalp: excision and coverage with free tissue transfer. Annals of plastic surgery. 1990 Jan;24(1):75-9..
7. de Varebeke SJ, De Schepper A, Hauben E, Declau F, Van Marck E, Van de Heyning PH. Subcutaneous diffuse neurofibroma of the neck: a case report. The Journal of Laryngology \& Otology. 1996 Feb;110(2):182-4.

8. Hoffman WY, Baker DC. Pediatric tumors of the head and neck. Plastic Surgery. 1990;5:3175..

9. Adkins JC, Ravitch MM. The operative management of von Recklinghausen's neurofibromatosis in children, with special reference to lesions of the head and neck. Surgery. 1977 Sep 1;82(3):342-8.

10. Zanella FE, Mödder U, Benz-Bohm G, Thun F. Neurofibromatosis in childhood. Computed tomographic findings in the skull and neck areas. RoFo: Fortschritte auf dem Gebiete der Rontgenstrahlen und der Nuklearmedizin. 1984 Nov; 141(5):498-504.

11. Huang GS, Huang CW, Lee HS, Chang WC, Lee $\mathrm{CH}$, Leu NH, Chen CY. Diffuse neurofibroma of the arm: MR characteristics. American Journal of Roentgenology. 2005 May;184(5):1711-2.

12. Ergün SS, Emel E, Aydn IH, Karabekir S, Büyükbabani N. Extracranial diffuse neurofibroma with intracranial extension. Plastic and reconstructive surgery. 2000 Feb 1;105(2):801. 